\title{
Symmetric instability of cross-stream varying currents
}

\author{
by Joseph Pedlosky ${ }^{1}$
}

\begin{abstract}
The symmetric instability of a simple shear flow in which the velocity is a linear function of the vertical coordinate but which varies slowly in the cross-stream direction is studied using an asymptotic analytical method. Explicit analytical solutions are found for the evolution of the envelope of the developing linear instability. Although the problem with no lateral variation yields cell-like instabilities growing in place, the lateral variation of the shear produces time dependence and crossstream propagation of the envelope and accompanying cells.

A similar solution is derived for the case of laterally uniform shear in a current whose depth slowly varies exponentially in the cross-stream direction producing similar time dependence to the otherwise stationary cell pattern.
\end{abstract}

\section{Introduction}

The phenomenon of the symmetric instability of a vertically sheared current - that is, an instability independent of the down-stream direction, is of interest in both atmospheric and oceanic contexts, as described in Stone (1966), Emmanuel (1979) for the former, and Allen and Newberger (1998) for the latter. Further references can be found in each of these cited papers.

The treatment of the problem has tended to fall into two distinct categories: either the treatment considers extremely idealized flows, for example a shear flow in the vertical $(z)$ direction and independent of the cross stream direction, or numerical modeling of more realistic flows. Given the importance of the process it seems useful to have an explicit analytical solution for a case in which the shear flow, while simpler than a realistic oceanic or atmospheric flow, does allow the consideration of non-trivial cross stream variations. That is the subject of the present study.

In the case where the flow occupies a layer of uniform depth $D$ but for which the vertical shear varies in the cross-stream direction, an explicit analytical solution demonstrates the reintroduction of time dependence to the inviscid, linear instability problem which otherwise predicts spatially fixed cells growing exponentially in time. The cross-stream variation of shear produces propagation of the cells in the cross-stream direction. A similar result occurs

1. Woods Hole Oceanographic Institution, Woods Hole, MA 02543 USA.email: jpedlosky@whoi.edu 
for a case where the shear is laterally uniform but the depth increases exponentially in the cross-stream direction.

In Section 2 the basic model is introduced and describes the basic asymptotic analysis while Section 3 presents the solution and results for a specific flow profile. Section 4 describes the analysis for the case in which the depth, $D$, varies in the cross-stream direction. In Section 5 the results are reviewed and discussed.

\section{The model and asymptotics}

Consider a basic state flow whose velocity in the $y$ direction, $V$, is a linear function of the vertical coordinate, $z$, and a slow function of $x$, the cross-stream coordinate. In this study I will consider a Boussinesq, ideal fluid, with buoyancy frequency $N$ rotating around the vertical axis providing a constant Coriolis parameter, $f$. The fluid is contained in a layer of depth $D$ and the velocity $V$ has the form

$$
V=V_{z}(z+D),-D \leq z \leq 0,
$$

where $V_{z}(x)$ is the vertical shear and is independent of $z$ but will be a slow function of $x$. The basic flow is in geostrophic and hydrostatic balance so that the shear is related to the horizontal density gradient,

$$
\rho_{o} f V_{z}=-g \frac{\partial \bar{\rho}}{\partial x} .
$$

Here, $\rho_{o}$ is the constant Boussinesq mean density while $\bar{\rho}$ is the laterally variable part of the basic state density field.

The linear equations for the perturbation velocities $(u, v, w)$, the perturbation density $\rho$, and the perturbation pressure, $p$ then become, assuming the hydrostatic approximation and the fact that the perturbations are independent of $y$, the down-stream coordinate, where subscripts denote differentiation.

$$
\begin{aligned}
& u_{t}-f v=-\frac{1}{\rho_{o}} p_{x}, \\
& v_{t}+u V_{x}+w V_{z}+f u=0, \\
& 0=-p_{z}-\rho g, \\
& \rho_{t}-\rho_{o} u f V_{z} / g-\rho_{o} w N^{2} / g=0
\end{aligned}
$$

The thermal wind balance has been used in (2.3d) to express the cross-stream density gradient in terms of the vertical shear (2.2). 
The condition of mass conservation for perturbations independent of the downstream coordinate, $y$, is satisfied by the representation of the velocity components $u$ and $v$ in terms of a stream function,

$$
\begin{aligned}
u & =-\psi_{z}, \\
w & =\psi_{x} .
\end{aligned}
$$

All variables can be eliminated in terms of the stream function leading to the governing linear equation,

$$
\left(\frac{\partial^{2}}{\partial t^{2}}+f\left(f+V_{x}\right)\right) \psi_{z z}+N^{2} \psi_{x x}-2 f \psi_{x z} V_{z}-f \psi_{x} V_{z z}=0
$$

Since the basic state velocity, $V$, is a linear function of $z$, the last term on the left hand side of (2.5) vanishes. The boundary conditions for (2.5) are

$$
\begin{aligned}
\psi & =0, \\
z & =0,-D .
\end{aligned}
$$

If $V$ were independent of $x$ the solution for $\psi$ would vary on a lateral scale on the order of $D$ (if a bit longer than $D$ to respect the hydrostatic approximation). In fact, the completely inviscid problem has its maximum growth rate for infinite horizontal wavenumber but such small scales would be damped by viscosity. Since for simplicity we are not treating friction directly, we will simply choose a realistic lateral scale for the individual cells to be of order of the depth or a bit greater. In fact, the approach to the maximum growth rate at infinite $k$ occurs rapidly as a function of wavenumber and so the growth rate is relatively insensitive to $k$ for scales on the order of the depth.

Now, when $V$ is a slowly varying function of $x$, the stream function $\psi$ will be expected to vary on the relatively fast $x$ scale, of order $D$, as well as the slow scale which is much larger than $D$. We exploit that fact by explicitly writing $\psi$ as a function of the fast variable $x$ and the slow variable $X$ where $X=\varepsilon x$ in which $\varepsilon$ is the slowness parameter and is the ratio of $D$ to the long scale in $x$ over which $V$ changes. Finally, as in the standard WentzelKramers-Brillouin (WKB) formulation, it is also necessary for the expansion that follows below to introduce a new fast scale defined by,

$$
\frac{\partial \xi}{\partial x}=s(X)
$$

where the function $s(X)$ must be determined by the analysis. This leads to the substitution for each $x$ derivative,

$$
\frac{\partial}{\partial x} \rightarrow s \frac{\partial}{\partial \xi}+\varepsilon \frac{\partial}{\partial X} .
$$


Similarly, we introduce a slow evolution time $T=\varepsilon t$ and in analogy with (2.8) write

$$
\frac{\partial}{\partial t} \rightarrow \frac{\partial}{\partial t}+\varepsilon \frac{\partial}{\partial T} .
$$

Since there is no explicit dependence on time of the basic state a new fast time variable is not needed. With the use of the new time and space variables we can rewrite (2.5) as

$$
\begin{aligned}
\psi_{z z t t} & +f^{2} \psi_{z z}+N^{2} s^{2} \psi_{\xi \xi}-2 f s V_{z} \psi_{\xi z} \\
= & -2 \varepsilon \psi_{z z t T}-\varepsilon^{2} \psi_{z z T T}-N^{2} \varepsilon\left\{2 s \psi_{\xi X}+s_{X} \psi_{\xi}+\varepsilon \psi_{X X}\right\} \\
& -\varepsilon \psi_{z z} f V_{x}+2 \varepsilon f \psi_{X z} V_{z} .
\end{aligned}
$$

An expansion in powers of $\varepsilon$ leads to the lowest order problem for $\psi^{(0)}$ for which the terms on the right hand side don't appear. The solution for the lowest order stream function that satisfies the boundary conditions (2.6) is then:

$$
\psi^{0}=A(X, T) e^{i k \xi+\sigma t} e^{i k s f V_{z} z /\left(\sigma^{2}+f^{2}\right)} \sin m z, m=n \pi / D .
$$

Here $k$ is the wavenumber in the new fast variable, $\sigma$ is the linear growth rate, and $n$ is the vertical mode number. It is understood that the real part of the expression in (2.11) is taken. The envelope $A(X, T)$ is a function of the slow space scale reflecting the slow variation of $V$ and the goal of the calculation is to obtain an evolution equation for $A$ on the slow time and space scales. Note that $V_{z}$ in the exponential term in (2.11) is a function of $X$. Since the coefficients of the lowest order problem are functions only of space and not of time, normal mode solutions are found with a constant $\sigma$.

The method is standard. The $O(1)$ solution is substituted into the $O(\varepsilon)$ terms on the right hand side of (2.10). Those inhomogeneous terms for the problem for $\psi^{(1)}$ generally contain secular forcing terms that would render our expansion invalid. To prevent that, the condition that must be used is that the forcing at this next order must be orthogonal to the operator on the left hand side for $\psi^{(1)}$. To order $\varepsilon$ that condition will yield the desired evolution equation for $A$. Before doing so, we need to use the fact that the solution (2.11) implies a dispersion relation between the growth rate $\sigma$ and the wavenumber $k$. That dispersion relation may be conveniently written

$$
\frac{f^{2} V_{z}^{2}}{\left(\sigma^{2}+f^{2}\right)}=\frac{N^{2}}{\sigma^{2}+f^{2}}+\frac{n^{2} \pi^{2} / D^{2}}{k^{2} s^{2}} .
$$

Note that for instability (i.e. $\sigma^{2}>0$ ) so we must have $V_{z} / N>1$.

Also, (2.12a) may be rewritten in a manner that will subsequently be very helpful, as

$$
k s=\frac{m\left(\sigma^{2}+f^{2}\right) / f}{\left(V_{z}^{2}-N^{2}\left\{\sigma^{2} / f^{2}+1\right\}\right)^{1 / 2}}
$$


or, equivalently, to show the explicit dependence of growth rate on wavenumber,

$$
\begin{aligned}
\sigma^{2} & =-f^{2}-k^{2} s^{2} \frac{N^{2} k^{2} s^{2}}{2 m^{2}} \\
& \pm \frac{\left\{k^{4} s^{4} N^{4} / m^{4}+4 f^{2} k^{2} s^{2} V_{z}^{2} / m^{2}\right\}^{1 / 2}}{2} .
\end{aligned}
$$

As mentioned above, for the classical problem in which the shear is independent of horizontal position the function $s$ just becomes a constant (that is, unity). Then the growth rate, formally, reaches its maximum value as $k$ becomes infinite although the limit is closely approached whenever $k D$ is greater than one. Very small lateral scales would be quenched by viscosity, which is not considered in this treatment, and instead we will just choose a realistic scale of order the depth in a range of $k$ where the growth rate curve is relatively flat with respect to $k$ in order to examine the effect of lateral variations of the shear.

The condition that $\sigma$ is a constant imposes a condition on $s(X)$ that must hold as either $V_{z}$ or $D$ is considered a function of the long space variable, $X$. The growth rate in (2.12) can be chosen as the growth rate for a particular value of the vertical shear (for example its maximum) and then (2.12) describes how the wavenumber, $k s$, changes with $X$ to keep $\sigma$ constant. For decreasing shear, for example, the wavenumber must increase to keep the $\sigma$ constant. The constancy of the growth rate is a standard WKB result analogous to the constancy of the frequency of a wave propagating through a variable medium that is independent of time. In both examples the constancy of the growth rate or frequency is required to keep the problem's solution from becoming secular with time and rendering the expansion in powers of $\varepsilon$ invalid.

The equation for the next order stream function in the $\varepsilon$ expansion is (2.10) with operator on the left hand acting on $\psi^{(1)}$ while the inhomogeneous terms on the right hand side are evaluated using the lowest order solution (2.11). To keep the expansion valid the forcing term must be orthogonal to solutions of the homogeneous operator of the left hand side. This condition can be written symbolically, after solutions for $\psi^{(1)}$ in the form are assumed, $\sim e^{\sigma t+i k s \xi} \Phi(X, T, z)$ as

$$
\int_{0}^{D} e^{-i k s f V_{z} z /\left(\sigma^{2}+f^{2}\right)} \sin m z\{\text { Right hand side }\} \mathrm{dz}=0,
$$

Note that the function with which the right-hand side is integrated is the complex conjugate of the lowest order stream function.

The result of carrying out the step demanded by (2.13) is straightforward but tedious with a great deal of algebra. It results, for the case where $D$ is constant and $V_{z}$ is independent of $z$ but a function of $X$, in the evolution equation for $A(X, T)$. After frequent use is made of the dispersion relation (2.12) we finally obtain,

$$
a_{1} \frac{\partial A}{\partial T}+a_{2} i \frac{\partial A}{\partial X}+a_{3} A=0
$$




$$
\begin{gathered}
a_{1}=\sigma\left(m^{2}+\frac{k^{2} s^{2} f^{2} V^{2}}{\left(\sigma^{2}+f^{2}\right)^{2}},\right) \\
a_{2}=\frac{m^{2}\left(\sigma^{2}+f^{2}\right)}{k s}, \\
a_{3}=-\frac{m^{2} f D}{2 k s} \frac{\partial\left(k s V_{z}\right)}{\partial X}+f V_{z X} \frac{D}{4}\left\{m^{2}+\frac{k^{2} s^{2} f^{2} V^{2}}{\left(\sigma^{2}+f^{2}\right)^{2}}\right\}+i \frac{(k s) f^{2} V_{z} V_{z X}}{2\left(\sigma^{2}+f^{2}\right)} .
\end{gathered}
$$

This linear partial differential equation has coefficients that depend on the slow space variable $X$ and the qualitative nature of the solution depends on that variation. It is first important to note that each term in the coefficient $a_{3}$, the coefficient of the third term, depends explicitly on the variation of the shear with cross-stream direction. If the shear were independent of $X$ the third term would vanish and the first two terms would have constant coefficients. In that case the solution of the first-order partial differential equation would simply reveal the change in growth rate for a small change in the wavenumber in the dispersion relation. The essence of the problem when there is lateral variation derives from the third term in (2.14a). It is through that term that interesting slow time dependence occurs.

\section{The solution for variable shear}

To obtain the solution to (2.14) when the shear is a function of $X$ it is useful to write

$$
A=B(X, T) e^{\varphi(X)} .
$$

When this form is used in (2.14) we first choose $\varphi$ to satisfy

$$
i \frac{\partial \varphi}{\partial X}=-\frac{a_{3}}{a_{2}} .
$$

Note that the right hand side of (3.2) is only a function of $X$. Furthermore, since each term in $a_{3}$ is proportional to $\frac{\partial V_{z}}{\partial X},(3.2)$ can be integrated directly resulting in an explicit expression for $\varphi$. After repeated simplification using the dispersion relation and (2.12b) we obtain

$$
\varphi=-i \frac{3}{4} m D \frac{V_{z} / a}{\left(V_{z}^{2} / a^{2}-1\right)^{1 / 2}}+i m D \ln \left(V_{z} / a+\left(V_{z}^{2} / a^{2}-1\right)^{1 / 2}\right)-\frac{1}{4} \ln \left(V_{z}^{2} / a^{2}-1\right)^{1 / 2}
$$

where

$$
a^{2}=N^{2}\left(\sigma^{2}+f^{2}\right) / f^{2} .
$$

This leaves the equation for $B(X, T)$,

$$
a_{1} \frac{\partial B}{\partial T}+i a_{2} \frac{\partial B}{\partial X}=0
$$


Note that $a_{1}$ are $a_{2}$ functions of $X$ (but not $T$ ). The equation for $B$ has the solution,

$$
\begin{aligned}
& B=B(\gamma(X)-i T), \\
& \frac{d \gamma}{d X}=\frac{a_{1}}{a_{2}}=\frac{\sigma m}{f}\left[\frac{1}{\left(V_{z}^{2} / a^{2}-1\right)^{1 / 2}}+\frac{V_{z}^{2}}{\left(V_{z}^{2} / a^{2}-1\right)^{3 / 2}}\right]
\end{aligned}
$$

so that the problem is reduced to the quadrature indicated by ( $3.5 \mathrm{~b})$. Note that if the shear were independent of $X$ the argument of $B$ would be linear in $X$ and $T$ reflecting the fact that a small change in wavenumber would lead to a small change in growth rate. Let's suppose the quadrature has been done (we will give an example shortly). Then the total solution can be written

$$
A(X, T)=B(\gamma(X)-i T) e^{\varphi(X)}
$$

The remaining step is to determine the function $B$. Suppose that at $T=0$ the initial condition is $A=A_{0}(X)$. For concreteness, consider the case where we imagine the initial linear disturbance amplitude to be independent of $X$ and inquire how the variation of the shear with $X$ will affect the subsequent structural development of the instability. Hence, if $A_{o}$ is independent of $X$ the initial condition yields,

$$
B(\gamma)=A_{0} e^{-\varphi(X)}
$$

To determine $B$ we write $X$ as a function of $\gamma$ by inverting the solution of (3.5b) to write $X=\hat{X}(\gamma)$ so that $B(\gamma)=A_{0} e^{-\varphi(\hat{X}(\gamma))}$ so that

$$
B(\gamma-i T)=A_{0} e^{-\varphi(\hat{X}[\gamma-i T])},
$$

with $\gamma$ given by (3.5b) as a function of $X$. Thus,

$$
A=A_{0} e^{\varphi(X)} e^{-\varphi(\hat{X}[\gamma-i T])}
$$

and where we note that $\gamma$ is a function of $X$.

Before considering a realistic, if idealized, example it is helpful to our understanding to consider a model situation to anticipate the nature of the space and time dependences for A that can arise. Suppose for example that our calculation of $\varphi$ and $\gamma$ yields the relations

$$
\begin{aligned}
& \varphi=\alpha\left(X / X_{o}\right), \\
& \gamma=\frac{\beta}{\sigma}\left(\frac{X}{X_{o}}\right)^{1 / 2},
\end{aligned}
$$


where $\alpha$ and $\beta$ are dimensionless constants (note that $\gamma$ has the dimensions of time). The inversion of the second relation yields $X / X_{o}=\left(\frac{\sigma \gamma}{\beta}\right)^{2}$ so that

$$
B(\gamma)=A_{0} e^{-\frac{\alpha \sigma^{2}}{\beta^{2}} \gamma^{2}}
$$

and a little algebra yields the solution

$$
A=A_{0} e^{i \frac{\sigma T}{\beta}\left(X / X_{o}\right)^{1 / 2}+\frac{\alpha \sigma^{2} T^{2}}{\beta^{2}}}
$$

which illustrates how a propagation of the amplitude envelope can arise. Note that the phase of $A$ moves satisfying $X \sim \frac{\text { const }}{T^{2}}$. Thus, our expectation is that a nontrivial variation in shear will result in (3.9) leading to an amplitude dynamics involving propagation. One way of interpreting this behavior is that although the basic solution has a single wavenumber, the slow variations in $X$ of the shear excite other modes with wavenumbers close to $k$ in the solution for $B$.

As a more physically plausible although more complex example, consider

$$
V_{z}^{2}=a^{2}+\frac{S^{2}}{(X / l)^{2}}
$$

where $a$ is given by (3.3b). The shear decays algebraically with the slow space scale $X$ where the small slowness parameter on which our asymptotics is based is $D / l$.

This form allows an easy evaluation of $\gamma(X)$-that is,

$$
\gamma=\frac{\sigma m}{f S l}\left[X^{2}+\frac{a^{2} X^{4}}{4 S^{2} l^{2}}\right]
$$

whose inversion yields

$$
X=\hat{X}(\gamma)=\left[\frac{2 S^{2} l^{2}}{a^{2}}\left\{-1+\frac{\gamma f a^{2}}{\sigma S l m}\right\}^{1 / 2}\right]^{1 / 2} .
$$

The function $\varphi$ is given directly by (3.3a) with (3.13) with the solution for $A$ following as

$$
A=A_{o} e^{\varphi(X)} e^{-\Phi(\gamma-i T)}
$$

where

$$
\Phi(\gamma-i T)=\varphi(\hat{X}(\gamma(X)-i T)
$$

Where in (3.17) the argument on the right hand side is the function $\hat{X}$ as in (3.15) in which, as indicated by ((3.17) $\gamma$ is replaced by $\gamma(X)-i T$. 


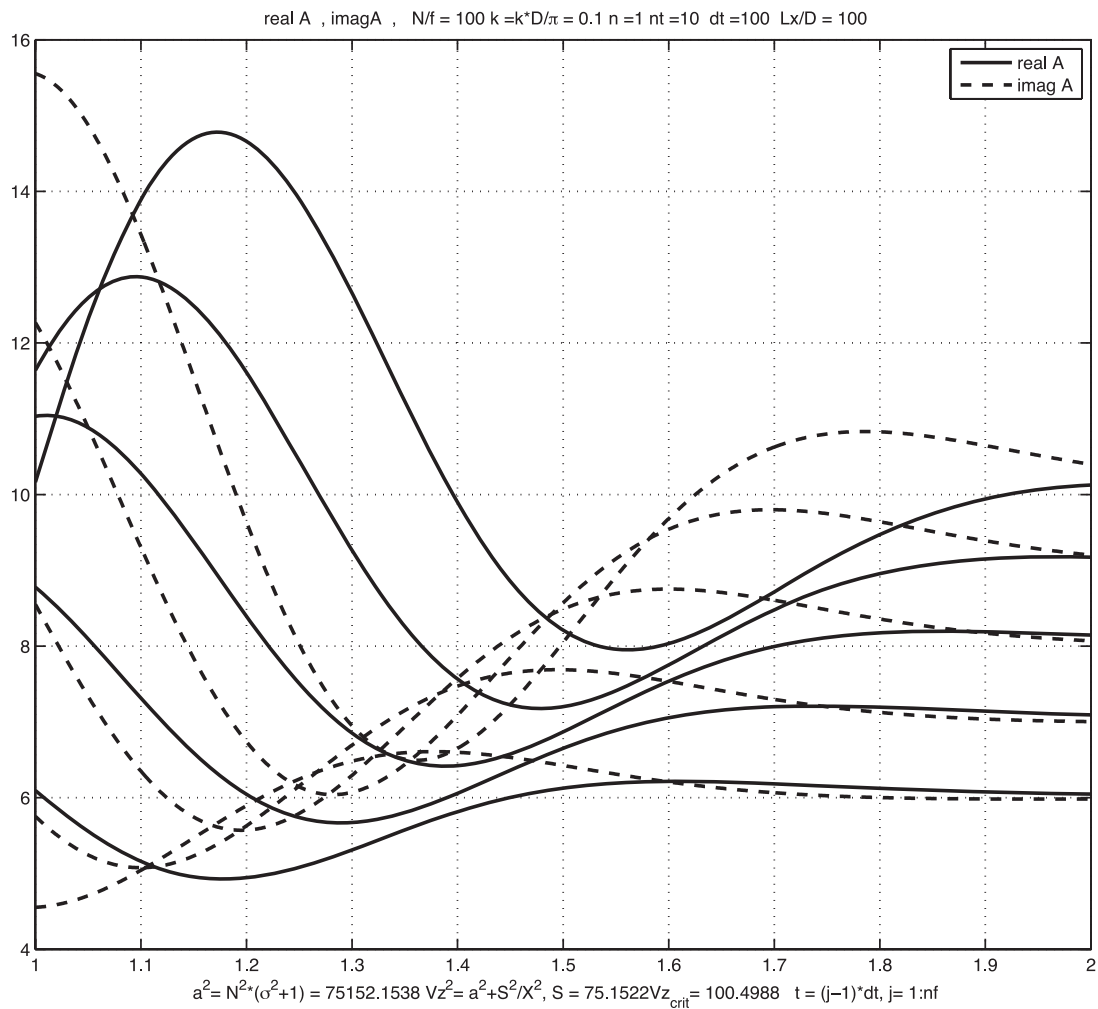

Figure 1. The amplitude $A$ as a function of $X$ for 5 time intervals. The larger amplitudes correspond to sequentially layer times separated by a $d T=100$. Note the progression of the peaks of both real and imaginary parts of $A$ to greater $x$; that is, toward weaker vertical shear although the growth of the amplitude is greater in the region of strong shear.

The solution is then complete. At the same time (2.7) and (2.12b) yields the variation in the wavenumber of the basic disturbance, i.e.

$$
k s=\frac{n \pi\left(\sigma^{2}+f^{2}\right)}{f S D}\left(X^{2}-X_{0}^{2}\right)
$$

where $X_{o}$ is an arbitrary constant. We will choose that constant to represent the smallest value of $X$ in the following calculations.

In the following figures $\sigma, N, V_{z}$ are nondimensional versions of the same variables but scaled with $f$. The wavenumber is scaled with $D / \pi$ and time is also scaled with $f$ while $X$ is scaled with $l$ such that $D / l<<1$.

Figure 1 shows the real and imaginary parts of $A$ for a value of $N / f=100$, and $\mathrm{kD} / \pi=0.1$ at 5 times separated by a long time interval of 10 . The curves are offset by a unit value at each time so that the higher curves are at progressively later times. The curves 


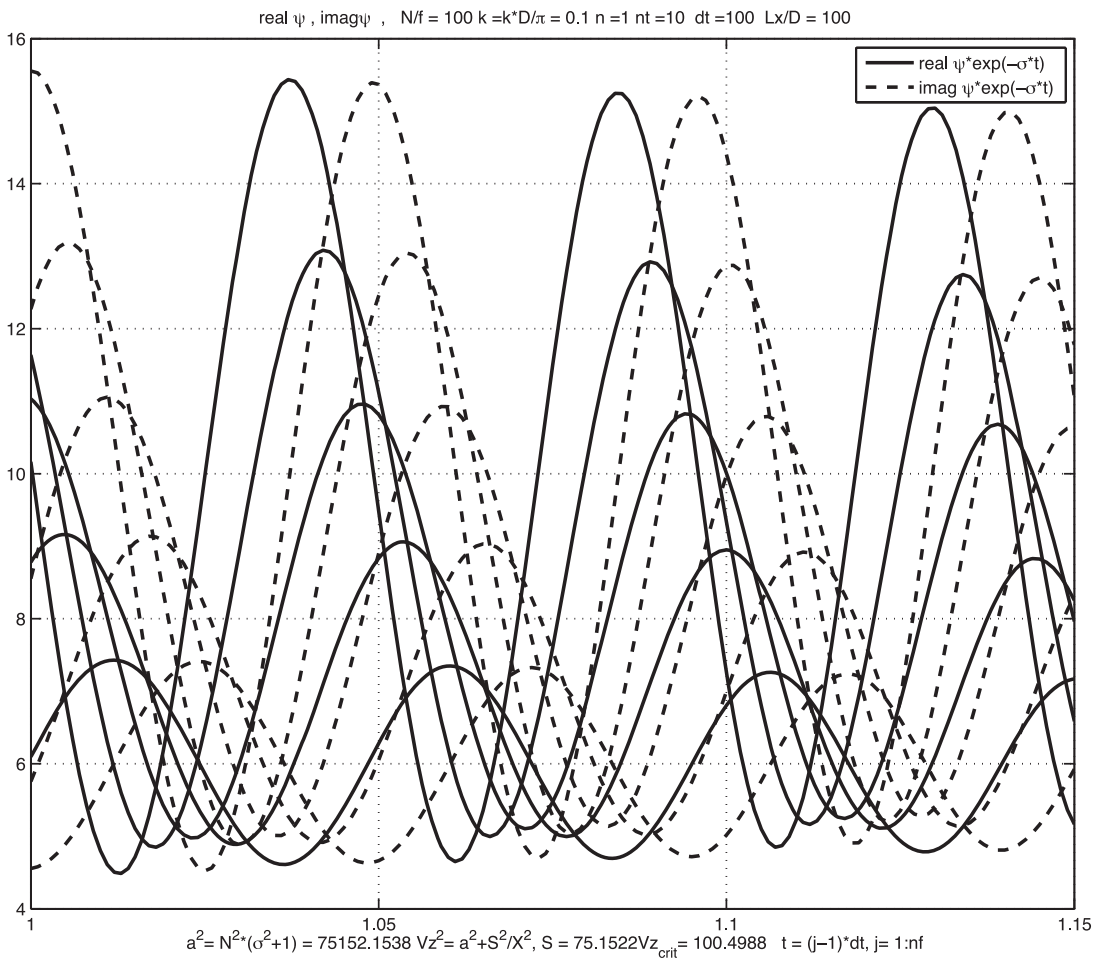

Figure 2. As in Figure 1 except that it is the real and imaginary parts of $A e^{i k \xi}$ that are plotted; that is, the lateral form of the streamfunction. Five time snapshots are shown for the same times as the times in Figure 1. Note, now, the total phase propagation towards larger shear.

of $A(X)$ demonstrate an evolution which favors growth in the region of high shear but keep in mind that the linear growth rate for the mode on the order one time scale is independent of space. It is interesting to note that the peak of the amplitude slowly progresses towards larger $X$-that is, weaker shear. This is a consequence of what might be called the distorted development resulting from the variation of the shear. To get a sense of the progression of individual cells of the instability we need to examine the shape of the stream function, which is proportional to $A e^{i k \xi}$, where $\xi$ is given by (3.18). The result for the amplitude is given in Figure 2 where the real and imaginary parts of $A e^{i k \xi}$ are shown. Five time snapshots are shown at the times of Figure 1. Note that this is equivalent to scaling the stream function by its exponential factor $e^{\sigma t}$ to concentrate on the slow time evolution produced by the variable shear. Since the stream function varies on a more rapid space scale than the amplitude, a restricted region is shown near $X=1$. It is clear from this figure that the individual cells will propagate to the left, towards larger shear once the more rapid phase on the fast space scale, $\xi$ is considered. A similar result is obtained for other values of the parameters. For 
example, Figure 3 shows a similar behavior for weaker stratification, $N / f=10$. Since the potential vorticity (pv) for this flow can be written,

$$
q=\left(f+V_{x}\right) N^{2}-f V_{z}^{2},
$$

the direction of propagation is in the direction of more negative pv.

\section{The solution for variable depth}

We consider now the case where the vertical shear of the basic state, $V_{z}$, is constant but the depth of the lower boundary at $z=-D$ varies slowly with $X$. In particular the basic state velocity will have the form

$$
V(z, X)=V_{z}(z+D(X)) .
$$

The governing equation is still (2.10) and the lowest order solution is again,

$$
\psi^{0}=A(X, T) e^{i k \xi+\sigma t} e^{i k s f V_{z} z /\left(\sigma^{2}+f^{2}\right)} \sin m z, \quad m=n \pi / D
$$

but with $D$ a slowly varying function of the slow space variable $X$, the resulting equation for the evolution of the amplitude $A$ will differ. However, the process of finding the evolution equation is the same. The evaluation of the right hand side of (2.10) by using (4.1) in those terms will lead to a forcing term for the next order problem for the unstable eigenfunction. The elimination of possible resonant terms of that forcing will yield the desired evolution equation. First, though, it is important to note that the condition that $\sigma$ be a constant imposes a condition on the function $s(X)$-that is,

$$
k^{2} s^{2} D^{2}=\frac{n^{2} \pi^{2}}{\left(\frac{f^{2} V_{z}^{2}}{\left(\sigma^{2}+f^{2}\right)^{2}}-\frac{N^{2}}{\left(\sigma^{2}+f^{2}\right)}\right)}=\text { const } .
$$

so that $k s$ inversely varies with $D$, so that

$$
k s(X)=\frac{D S K}{D(X)}
$$

where $D S K$ is a constant which can be fixed by choosing the $s$ to be unity at some point, for example where the depth is a minimum in a model of a coastal region.

After a great deal of tedious but straightforward algebra the desired equation for $A$ is,

$$
\begin{aligned}
& \sigma\left(n^{2} \pi^{2}+\frac{D^{2} k^{2} s^{2} f^{2} V_{z}^{2}}{\left(\sigma^{2}+f^{2}\right)^{2}}\right) \frac{\partial A}{\partial T}+i \frac{\left(\sigma^{2} f^{2}\right) n^{2} \pi^{2}}{k s} \frac{\partial A}{\partial X} \\
& +A\left[f V_{z}\left\{k s D_{X}-D(k s)_{X}\right\}+\frac{D_{x} f V_{x}}{2}\left\{n^{2} \pi^{2}+\frac{(k s)^{2} D^{2} f^{2} V_{z}^{2}}{\left(\sigma^{2}+f^{2}\right)}\right\}\right]=0 .
\end{aligned}
$$




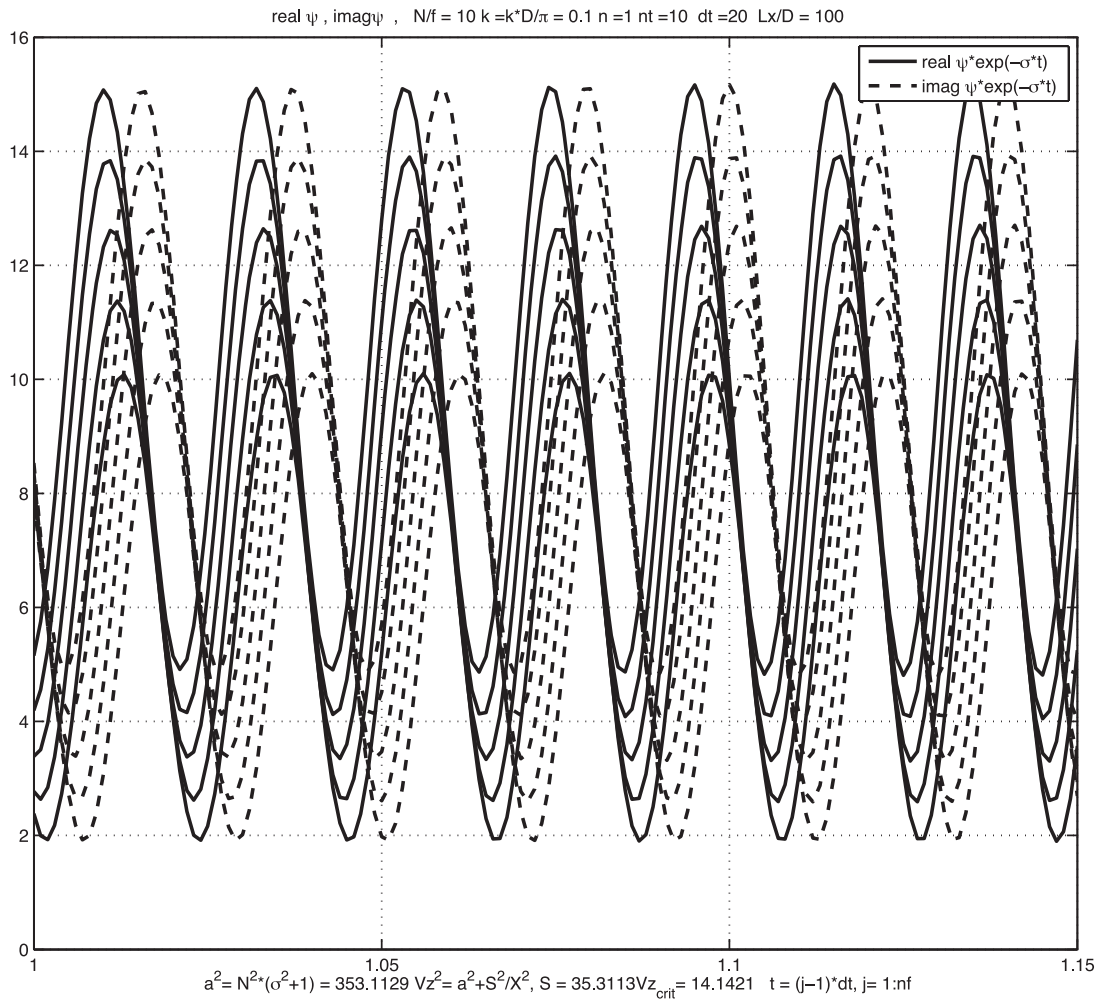

Figure 3. As in Figure 2 except that the stratification is 10 times smaller; that is, $N / f$ is 10 .

Note that the combination $k s D$ is constant by (4.4) and that the coefficient of $A$ in (4.5) is strictly real.

The solution for $A$ can be found in,

$$
A=B(X, T) e^{i \Phi(X)}
$$

whose form is a bit more convenient since the coefficient of $A$ is real. Using (4.4), this leads to

$$
\frac{\partial \Phi}{\partial X}=\frac{f V_{z} D K S}{\left(\sigma^{2}+f^{2}\right)} \frac{D_{X}}{D}\left(2+\left[\frac{1}{2 n^{2} \pi^{2}}\left\{n^{2} \pi^{2}+\frac{D K S^{2} f^{2} V_{z}^{2}}{\left(\sigma^{2}+f^{2}\right)}\right\}\right]\right)
$$

whose integral is

$$
\begin{aligned}
& \Phi=\mu \ln \left(D / D_{0}\right) \\
& \mu \equiv \frac{D K S f V}{\left(\sigma^{2}+f^{2}\right)}\left[3 / 2+\frac{1}{2} \frac{D K S^{2} f^{2} V_{z}^{2}}{\left(\sigma^{2}+f^{2}\right)^{2}}\right]
\end{aligned}
$$


while $B$ satisfies

$$
\sigma \frac{\partial B}{\partial T}\left(n^{2} \pi^{2}+\frac{D K S^{2} f^{2} V_{z}^{2}}{\left(\sigma^{2}+f^{2}\right)^{2}}\right)+\frac{i}{k s}\left(\sigma^{2}+f^{2}\right) n^{2} \pi^{2} \frac{\partial B}{\partial X}=0
$$

Again, solutions can be found in the form,

$$
\begin{aligned}
& B=B(\gamma(X)+i T), \\
& \gamma=-\int_{X_{o}}^{X} \frac{\beta}{D\left(X^{\prime}\right)} d X^{\prime}, \\
& \beta=\frac{\sigma}{\sigma^{2}+f^{2}}\left(1+\frac{D K S^{2} f^{2} v_{z}^{2}}{n^{2} \pi^{2}\left(\sigma^{2}+f^{2}\right)^{2}}\right)
\end{aligned}
$$

so that

$$
A=B(\gamma+i T) e^{i \mu \ln \left(D / D_{0}\right)}
$$

Note the change in sign of argument in (4.11) compared to (3.6), a change that is convenient for the current example.

The forms of (4.10) and (4.11) are so simple in this case, as opposed to the variable shear example that explicit solutions are rather easily obtained. For example, for

$$
D=D_{0} e^{\alpha X},
$$

which is not a bad model of the depth variation in a coastal region, the solution for an initial condition of uniform amplitude, $A_{0}$, is

$$
\begin{aligned}
\gamma & =\frac{\beta}{\alpha D_{0}} e^{-\alpha X}, \\
B(\gamma+i T) & =A_{0} e \int_{0}^{X} e^{i \mu \ln \left(\gamma\left(X^{\prime}\right)+i T\right)-i \mu \ln \left(\beta / \alpha D_{o}\right)} d x^{\prime}, \\
\Rightarrow \frac{A}{A_{0}} & =e^{i \mu \ln \left(1+i \frac{T \alpha D_{0}}{\beta} e^{\alpha X}\right)} .
\end{aligned}
$$

Note that the solution for the envelope remains constant on the trajectory

$$
\alpha X=-\ln (T)+\text { const } .
$$

or

$$
\alpha \frac{d X}{d T}=-\frac{1}{T}
$$




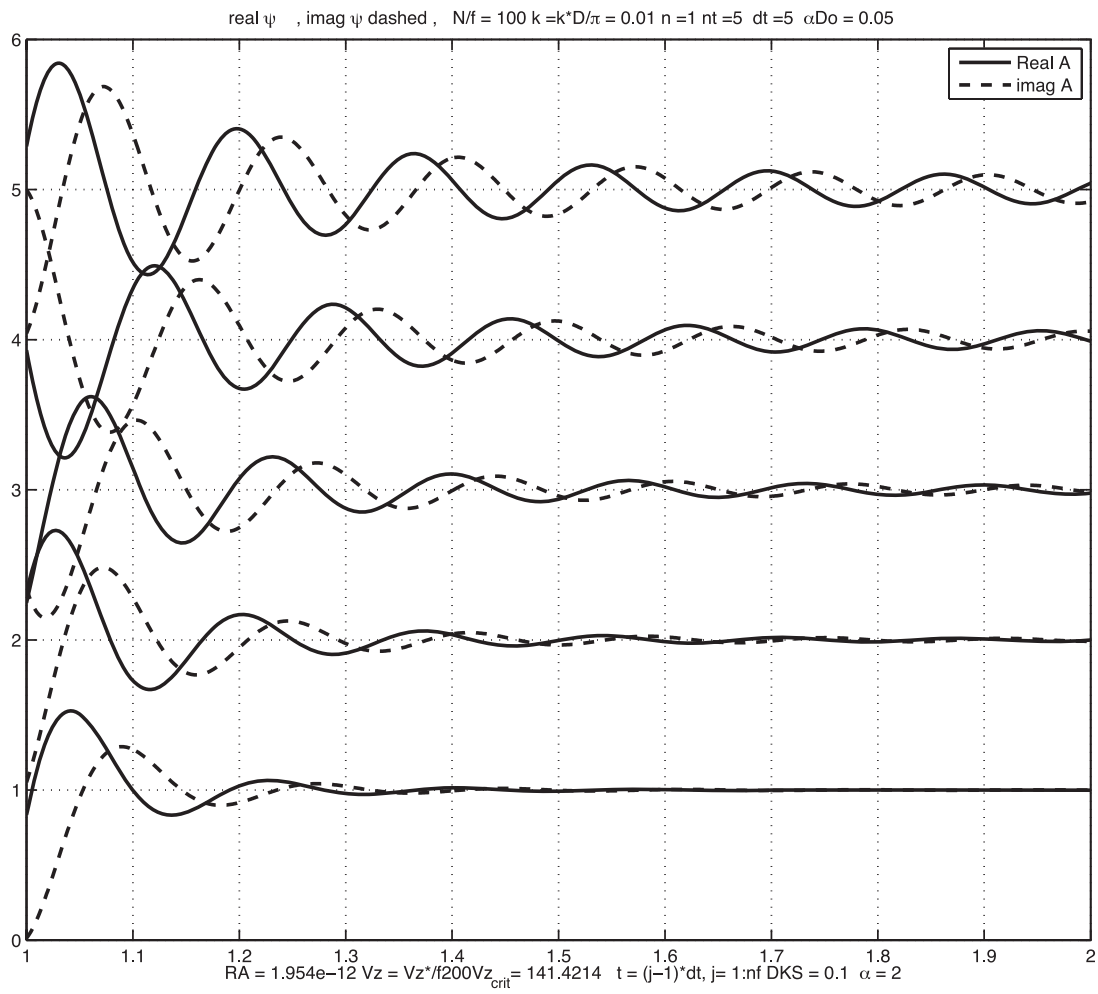

Figure 4. As in Figure 2 showing the real and imaginary parts of $A e^{i k \xi}$ for the lateral form of the streamfunction for variable $D=D_{o} e^{\alpha X}$. The smallness parameter is $\alpha D_{0}=0.05$. In this plot the figures are scaled by their maximum values and displaced by a unit amount vertically for each later time.

Thus, constant values of the envelope will move towards smaller $X$ values - that is, towards shallower water. For small values of $T$ (or small values of $\alpha$, a good approximation of (4.13) is

$$
\frac{A}{A_{0}}=e^{-\mu \frac{\alpha T D_{0}}{\beta} e^{\alpha X}+\frac{i}{2} \frac{\left(\mu \alpha T D_{0}\right)^{2}}{\beta^{2}} e^{2} \alpha X+\ldots}
$$

so that for a small time the phase of the solution is not affected. As time goes on the phase change will also produce a propagation of the cells as well as the amplitude to smaller depth. A typical example is shown in figure 4 where the amplitude, scaled by its maximum is plotted at four sequential times with the curves shifted vertically by a unit value for each subsequent time. 


\section{Discussion}

The theory presented above hinges on the slowness in the variation of the current shear or the fluid depth and hence it is difficult to apply the results directly to realistic coastal regions where symmetric instability is expected, as in Allen and Newberger (1998). The simple shear structure excludes the possibility of the importation of positive potential vorticity to stabilize the developing perturbation. However, in spite of its simplicity the model is useful in presenting a mechanism for the apparent propagation of amplitude and phase of the disturbances. This is especially provocative because an obvious mechanism for wave propagation is absent. Most of the temporal and spatial variation of the growing disturbance is due to what might be called "distorted development" arising from the solution of the function $B$ forced by the variation of properties of the flow as presented by the coefficient of $A$ in (2.14) and (4.5). The simple example of (3.10) through (3.12) is an illustration of the basic process.

One way to think about the process is to imagine a regular perturbation expansion without the introduction of slow time and space variables. Then the forcing terms on the right hand side of (2.10) are, except for the exponential in time factor of the lowest order stream function, just a function of the slow variation in $x$ of the shear. The forced solution could be found as a Fourier series in $x$ and the slowness of the variation of $V_{z}$ would yield wavenumbers in the neighborhood of the original wavenumber $k$. To satisfy initial conditions, a homogeneous solution with that range of wavenumbers needs to be found so that the sum of the homogenous solutions (each with slightly different $k$ and $\sigma$ ) and the forced solution satisfies the initial condition. This solution process is obviously clumsy and the equivalent is obtained more efficiently by the asymptotic analysis presented above; but it does suggest why the evolution of the full solution, with the interference and reinforcement of the various modes produced by the initial condition leads to what I have called distorted development.

The extension of these ideas to more realistic oceanographic flows would be of interest.

Acknowledgments. The author is grateful to Ken Brink for several helpful conversations during the development of this study and to an anonymous and conscientious reviewer who helped correct several minor errors.

\section{REFERENCES}

Allen, J. S. and P. A. Newberger. 1998. On symmetric instabilities in oceanic bottom boundary layers. J. Phys. Ocean., 28, 1131-1151.

Emanuel, K. A. 1970. Inertial instability and mesoscale convective systems. Part I: Linear theory of inertial instability in rotating viscous fluids. J. Atmos. Sci., 36, 2425-2449.

Stone, P. H. 1966. On non-geostrophic baroclinic instability. J. Atmos. Sci., 23, 390-400.

Received: 11 February 2014; revised: 11 April 2014. 